\title{
Automation of TEM Optical Alignments
}

Jan Jíša and Ondřej Svoboda

Thermo Fisher Scientific, Brno, Jihomoravsky kraj, Czech Republic

The TEM optical alignment automation module Align Genie is able to (fine-)tune the optics and column alignments in a robust, reliable and user-friendly way. Align Genie eases the learning curve for the novice operators, improves time-to-data for the experienced operator and is ideal for multi-user environments.

The first released version consist of the following fully automated functions in TEM mode: Find Beam, Center Beam, Focus Beam, Stigmate Beam, Center C2 Aperture, Align Pivot Points, and Focus Image. They can be run individually, sequentially and planned in advance for an unattended run. Additionally, Align Genie can automatically align and check several essential column alignments; it can center the beam between magnifications in HM sub-modes and also focus and stigmate the beam for individual spot sizes.

Align Genie consists of two user levels/tabs, one for access to the basic daily tuning routines for regular user login and the other with additional access to automated column alignments, optics checks and advanced settings for the supervisor user login.

The approach to algorithmization was to create a set of basic, generic building blocks with defined precondition checks and the ability to auto-calibrate. These were then hierarchically combined into longer alignment sequences. During the development, thousands of test runs in different conditions were performed and statistically evaluated to prove the required performance.

The building blocks use advanced image processing methods to identify the scene characteristics (illumination state, beam shape etc.), which are then abstracted and fed back to the alignment part of the algorithm. They are subsequently used in combination with experimentally gathered response models to align the microscope.

The user is optionally provided with a rich visual and text information about the progress. In case of a failure, this includes an attempt to identify possible causes.

The ultimate goal is to automate the complete column alignments and to reach the desired functionality in all conditions and optical modes (TEM/STEM, nanoprobe/microprobe, imaging/diffraction etc.) for all microscope configurations. 


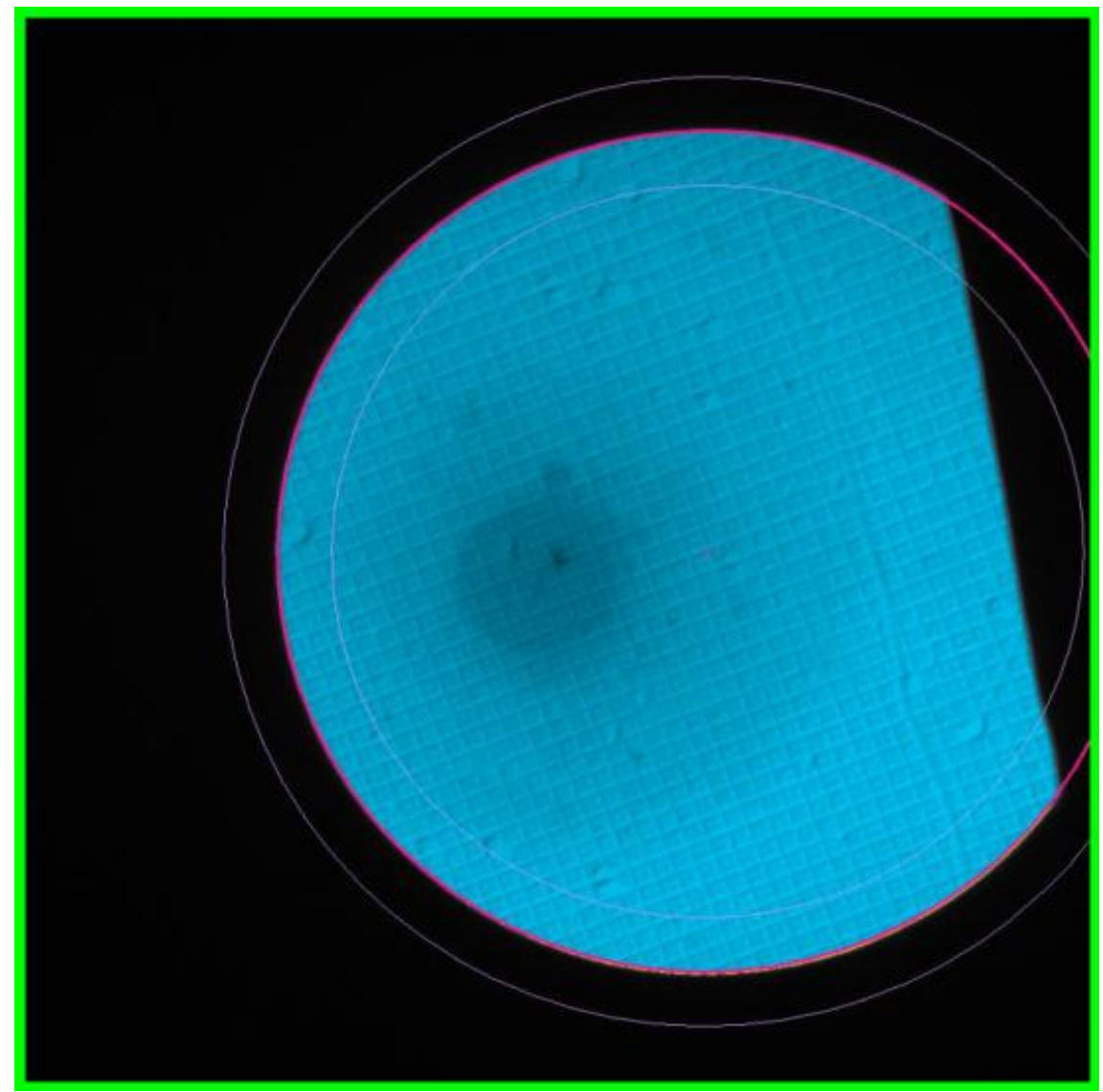

Figure 1. Example of image processing of a partially occluded beam.

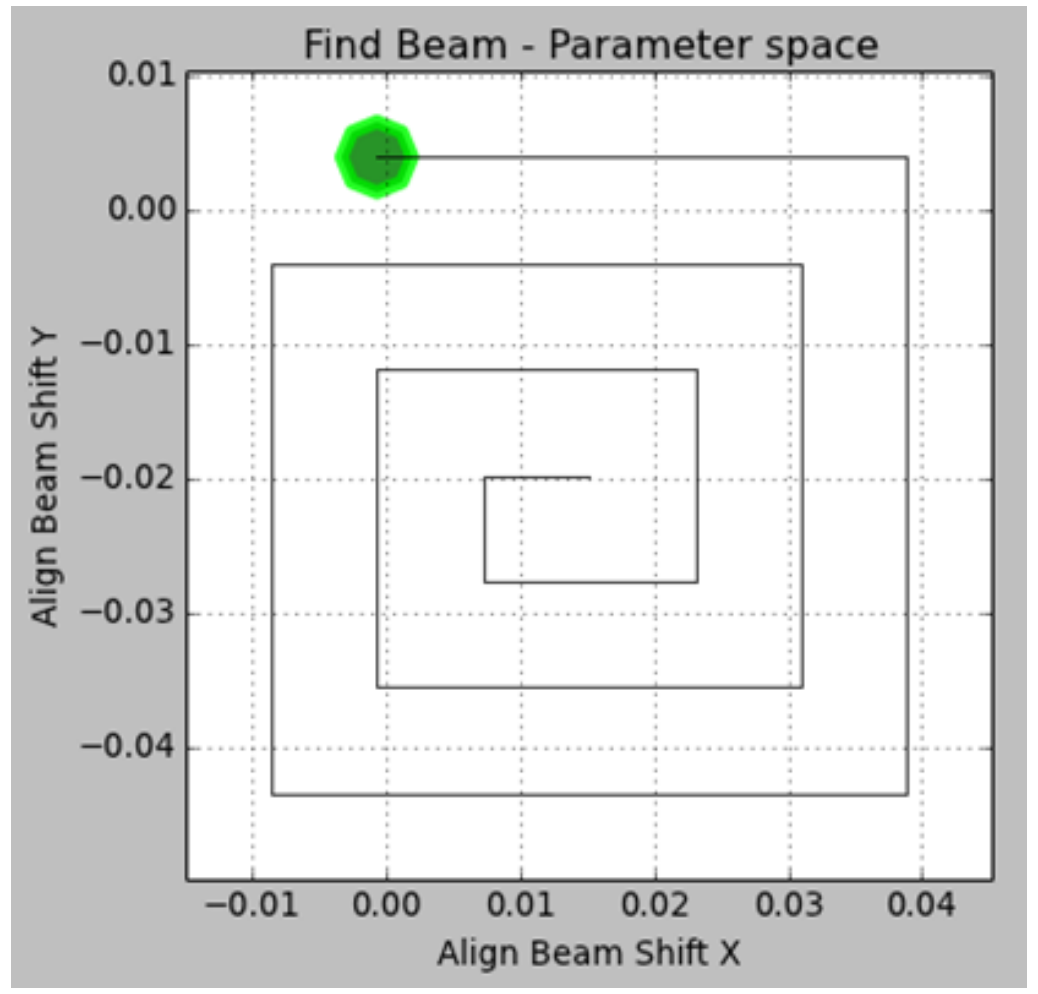

Figure 2. Find Beam algorithm visualization - spiral parameter space search. 